\title{
La escenificación en teatro como estrategia didáctica para la enseñanza de una segunda lengua "inglés""
}

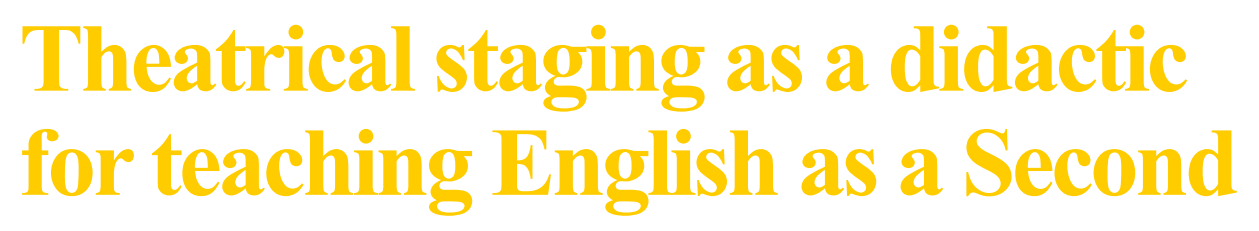
Language

\author{
Alejandro Bedoya ${ }^{2}$ \\ Astrid Ramírez Valencia ${ }^{3}$
}

\section{RESUMEN}

En el presente artículo se analiza la importancia de la enseñanza de una lengua extranjera y el diseño de metodologías didácticas en la ejecución y los presupuestos teóricos guía del English Day en el colegio Gimnasio los Alerces, de la ciudad de Bogotá. Así mismo, se muestran elementos para enseñar una lengua extranjera a

1 Este trabajo es un estudio de caso desde una propuesta didáctica desde la escenificación (teatro) y la importancia del aprendizaje del inglés para básica secundaria en Colegio Gimnasio Los Alerces, Bogotá. Para optar por el título de Licenciado en lenguaje extranjera con énfasis en inglés de la universidad Distrital Francisco José de Caldas, asesorado por la Dra. Astrid Ramírez Valencia.

$2 \quad$ Estudiante de la Licenciatura en lengua extranjera con énfasis en inglés, Docente de inglés con trayectoria en diversas instituciones privadas. correo alejandro. bedoya@gcrb.edu.co - Noviembre. 2020. ORDID: https:// orcid.org/0000-0002-6619-4923

3 Docente Universidad Distrital Francisco José de Caldas Bogotá, Colombia, Ph.D. en Lenguaje y cultura, formadora de profesores de inglés durante más de 25 años. Investigadora y maestra en la Universidad Distrital Francisco José de Caldas. correo aramirezv@udistrital.edu.co ORCID: https://orcid.org/0000-0002-3025-5982 través del teatro: se conceptualiza la modelación y escenificación como estrategia didáctica. Estos desde las experiencias didácticas desde los ámbitos escolares, dependiendo del lugar (docente, tradición, contexto, recursos) se presenta un modelo de programa que puede seguir la institución para transformar la enseñanza desde el teatro, como didáctica para la enseñanza eficiente de la lengua extranjera.

PALABRAS CLAVE: didáctica, escenificación, programa pedagógico, lengua extranjera.

\section{ABSTRACT}

This article analyzes the importance of teaching a foreign language and the design of didactic methodologies, in the execution and theoretical assumptions that guide the English Day at the Gimnasio los Alerces School, in the city of Bogotá. Additionally, elements are shown to teach a 
foreign language through theater: modeling and staging are conceptualized as a didactic strategy. These from the didactic experiences from the school environments, according to the place (teacher, tradition, context, resources) a program model is presented that the institution can follow to transform teaching from the theater, as didactics for the efficient teaching of a Foreign language.

KEYWORDS: didactics, staging, pedagogical program, foreign language.

\section{INTRODUCCIÓN}

Desde la lúdica como un campo de método de enseñanza dentro de la didáctica, en términos pedagógicos, se hace necesario que la lúdica se entienda dentro del marco de unos propósitos, con unas enseñanzas, unos métodos, unos procesos, unas secuencias prestablecidas, es así, que la presencia de estos recursos sea pertinente y marcan una manera adecuada de evaluación. Esto convirtiéndola en una estrategia lúdica particular que cuente con componentes curriculares claros, y se convierte en una didáctica pertinente con la que los estudiantes disfrutan, aprenden, resuelven conflictos, cooperan y sacan adelante proyectos. Tal es el caso del uso del teatro para la a enseñanza de la lengua extranjera (en adelante L2).

Sin un propósito claro en el campo de la lúdica o didácticas de la enseñanza de la $L 2$, no podría haber una intervención pedagógica transformadora; y el alcance de los resultados pueden ser bajos, puesto que, en muchas ocasiones, sus derivaciones son el producto de una mirada externa al contexto real por cuanto se hace por fuera de situación, un ejemplo son la enseñanza de estructuras gramaticales no solo desmotiva, sino que se hace injustificable desde el punto de vista de la mediación cultural; la gramática es una herramienta con la que se puede entender los aspectos de una lengua, donde su utilidad solo es en el aspecto formal, no en su completa aplicación, la enseñanza en el inglés aun cae en el cliché de algunos profesores, que no avanzan del verbo to be, se debe a ello: que enseñan formas verbales sin un contexto claro, en donde haya una situación comunicativa que amerite el uso de las formas verbales; como no hay contexto, la enseñanza del verbo llega a ser instrumento cognitivo para resolver un problema o una necesidad comunicativa, y se queda como contenido de un aprendizaje memorístico, anquilosado, aburrido y que se olvida a mediano plazo.

De esta forma, se puede poner al teatro como una evidencia que reúne varios elementos de la lúdica y se pueden aprovechar para brindar un contexto pertinente al acto comunicativo, dicho contexto o situación no necesariamente debe tener el estatus de real (como cuando llegamos a un hotel en el extranjero y requerimos conocer ciertos códigos para entender y comunicar un mensaje), sino que el "juego" puede hacer las veces de sustituto, de simulador, para que los estudiantes se sumerjan en situaciones comunicativas que les cree la necesidad de códigos, vocabulario y estructuras, entre otras.

\section{JUSTIFICACIÓN}

La estrategia desde el teatro como didáctica de la L2, pone en evidencia una falta mayor rigor conceptual desde los contextos, es decir, que se apropien los procesos dentro de las instituciones que utilizan la lúdica como la enseñanza de L2, para sistematizar los puntos más fuertes de las experiencias documentadas y facilitar su distribución o replica; por otro lado, a fuerza de las particularidades de las instituciones, las experiencias sobre el uso del teatro deben tener énfasis diferentes: en algunos planteles educativos es posible que el docente destaque por habilidades histriónicas, por organización logística; en otras ocasiones la escogencia de la obra también debe de ser variacional; se puede tener en cuenta el nivel de lengua extranjera, además de otros recursos que cuenten las 
instituciones. Por ello, se hace necesario un diseño instruccional con los elementos para llevar esto a cabo la experiencia en el Colegio Gimnasio los Alerces, y se hizo al tiempo que la formulación de conceptualización del proceso de la práctica, evidenciándose en documentación y recolección de estos procesos, que pueden nutrir la sistematización instruccional, y haciéndose conveniente tener una guía para el diseño instruccional, para el uso de este proyecto en otros años lectivos.

\section{OBJETIVO}

- Diseño de una estrategia lúdico didáctica desde la escenificación(teatro) para la enseñanza de una lengua extranjera $L 2$.

\section{OBJETIVOS ESPECÍFICOS}

- Análisis conceptual dentro del teatro como lúdica, desde algunas didácticas de la lengua extranjera; posturas desde Estados Unidos, Francia, Rusia y Colombia.

- $\quad$ Evidenciar desde el análisis, descripción, participación y proceso didáctico dentro de la celebración del English Day en el Colegio Gimnasio Los Alerces en 2019, para alcanzar los propósitos pedagógicos de la enseñanza $L 2$, a través de la escenificación (teatro)

\section{METODOLOGÍA}

Este trabajo es de tipo mixto, puesto que se tiene en cuenta las etapas propuestas por Denzin y Lincoln (2015): el primero corresponde al hermenéutico (comprensión del contexto); el segundo, descriptivo; el tercero, argumentativo; y el cuarto, analítico. Esto permitiendo que se haga un abordaje completo del ámbito educativo, permitiendo conocer los procesos de la enseñanza L2 en los campos de saberes, y aplicados para la transformación de dicha lúdica, para alcanzar los objetivos estipulados; la investigación tiene, por un lado, un carácter documental (cuantitativo) en los términos de Díaz, Mendoza y Porras (2011) para la investigación educativa y en comunicación, dado que se extraen los conceptos fundamentales a partir de la revisión y análisis de un pequeño estado de arte, porque juegan un papel clave en la interpretación de resultados y en la propuesta propia; por otro lado, tiene también un carácter etnográfico, pues el autor es, a la vez, realizador de la experiencia. Por ello se utiliza dentro del English Day del 2019 del grado octavo, en el Colegio Gimnasio Los Alerces, con lo que también se enmarca en un estudio de caso que recoge las experiencias en torno a esa jornada.

\section{MARCO TEÓRICO:}

Concepción de la enseñanza de L2 a través del teatro en Estados Unidos, Francia, Rusia, Japón y Colombia: La investigación, Conceptualizing Drama in the Second Language Clasroom, fue elaborada por la doctora Kathleen Rose MacGovern (2017) en la Universidad de Georgia, Estados Unidos. Se trata de una valiosa conceptualización de las formas en que el teatro ha sido usado para la enseñanza de la segunda lengua. Según expone la autora los investigadores y docentes llamaban técnicas de drama/teatro/escenificación a cosas que, si bien estaban relacionadas, eran disímiles y traían diferentes consecuencias en el proceso de enseñanza aprendizaje.

Así pues, se documenta que hay dos grandes maneras de entender el teatro en la enseñanza de L2. La primera desde el enfoque audilingual, en el que sucede la repetición de un guión prestablecido, a la manera de un Hamlet, con el objetivo de desarrollar las competencias lingüísticas a través de la memorización y la evocación También contempla la escritura original de un guión por parte del grupo, para que el trabajo creativo origine lazos que sirven para 
un mejor ambiente, esto como una excelente estrategia para la enseñanza de la gramática, pues implica la cooperación, la ayuda de pares y formas creativas de memorización no rutinarias. Por otro lado, está el enfoque kinestésicocorporal, en donde se hace más énfasis en la puesta en escena y en el papel que tiene el cuerpo en el proceso de enseñanza-aprendizaje de L2, esta segunda concepción se acomoda más al llamado enfoque comunicativo, mientras que la primera está más cercana al papel que la gramática y la "correcta forma" juegan en el aprendizaje, por este motivo McGovern insiste en la necesidad de ser precisos al hablar de teatro en la enseñanza de L2.

Basada en estas dos concepciones, se señala que hay tres aproximaciones didácticas que se han explorado en su recorrido investigativo: la escritura de guión, los juegos de rol y la puesta en escena. En la primera, el grupo de estudiantes se ven implicados en la parte escrita del proceso, como oportunidad de que la gramática tome parte del proceso. La importancia de los juegos de rol estriba en que, a partir de unas condiciones, instrucciones y reglas iniciales que dependen del contexto del juego, haya lugar a la improvisación, la aparición de lo inesperado, lo imprevisto y la espontaneidad, que es un rasgo fundamental en las conversaciones reales, y que son un excelente ambiente para el aprendizaje. L La tercera consiste en la puesta en escena, es decir, la presentación de una obra de teatro en el sentido Shaksperiano de la palabra, con papeles, personajes, líneas propias en donde acaece una historia actuada.

De acuerdo con la historia de esta práctica para la enseñanza-aprendizaje de $L 2$, el primero en documentarla fue Richard Via (1972), quien viajó a Japón para enseñar inglés. Según McGovern, el propósito de Via al usar la Puesta en Escena era lograr la introducción de conceptos socioculturales propios de los contextos en donde se habla L2 como lengua materna. En pocas palabras, a través del cuerpo, es posible servirse del teatro para enseñar conceptos y habilidades contextuales propias de la visión de mundo de una cultura, lo que pasa inadvertido por la gramática. Dicho de otra manera, con el teatro se puede enseñar competencias pragmáticas de una lengua extranjera. Esto último da cabida a los enfoques sociolingüísticos e interculturales para la enseñanza de L2. Para finalizar, una de las ideas conclusias de McGovern es que la escenificación, en cualquiera de sus tres aproximaciones didácticas, revista al grupo de aprendizaje de más cosas que el aprendizaje de un L2: aprendizaje experiencial, construcción de identidad como sujetos performáticos, confianza y trabajo en equipo; así como también una especial predisposición a reconocer de forma abierta al otro: otras costumbres, hábitos, creencias y valores. La enseñanza conceptual es distinguir entre drama y teatro. Drama es toda actividad donde el estudiante tenga que representar dentro del aula algún tipo de experiencia. Así pues, hay tres variantes del drama en la enseñanza de L2.

La investigación Incorporating theatre techniques in the language classroom, de Abigail Paul (2015), da un panorama sobre el uso del teatro en la enseñanza de la lengua extranjera en Chicago. Ellos pertenecen a una fuerte tradición donde el teatro y la enseñanza han estado emparentados desde 1959, por medio de figuras como Augusto Boal, Viola Spolin, Keith Johnstone y la misma Abigail Paul. La forma en que Paul nos muestra y sistematiza sus experiencias tienen que ver con un contraste entre el enfoque comunicativo y la improvisación. Según ella, un rasgo esencial del enfoque comunicativo es la creación de situaciones "reales" del uso del lenguaje que le permita al aprendiz el desarrollo de la competencia comunicativa. Se trata de las situaciones típicas de un curso de lengua extranjera donde hay interacciones en contextos como el hotel, el aeropuerto, el museo, la oficina y la escuela, entre otros. 
Paul advierte que el teatro de improvisación cumple con la misma función de las situaciones vitales (por no decir "reales"), y le agrega un componente lúdico importante a la hora de la efectividad del aprendizaje. En Chicago esto ha sido desarrollado gracias a la filosofía que subyace a la práctica del teatro, según ellos, la actuación no es un talento de pocos sino algo inherente a todo ser humano, por lo cual la dicotomía espectadora/actor es artificiosa. Las técnicas del teatro están al alcance de todos porque todo ser humano tiene la capacidad de llevarlas a cabo y, más específicamente, beneficiarse de ellas incluso para el aprendizaje de una segunda lengua. Así es como nace el concepto de espectactor: el teatro improvisado crea lazos de amistad, disfrute y placer, lo que crea un ambiente propicio para unos de los objetivos didácticos: que los estudiantes estén expuestos a la mayor cantidad de errores posibles sin que ello implique penalizaciones o castigos. En este sentido, el error se utiliza él mismo como parte de la didáctica, al crear la situación para que se dé, con el objetivo de hacer una retroalimentación instantánea. Lo mejor de esto es que dicha retroalimentación no tiene que ser dada por el profesor, sino que las más de las veces se da de forma natural entre los mismos estudiantes. El rol del profesor es descrito así: "el reto del profesor o líder es activar a cada estudiante en el grupo respetando la inmediata capacidad de cada cual para la participación." (Spolin 1999, citado por Paul, 1995, 119). Cabe destacar que esto va en consonancia con las pedagogías activas que no consideran al profesor como garante de una verdad que entrega al estudiante, sino como una guía que le permite al estudiante explorar sus intereses. Las actividades que sugiere son juegos de rol y situaciones hipotéticas dentro de un ambiente que tenga en cuenta el nivel de vocabulario de los estudiantes, condición esencial para poder jugar. La conclusión es que usar el enfoque comunicativo unido a técnicas de improvisación, crea un mejor ambiente para la enseñanzaaprendizaje de la lengua extranjera.

Desde Francia, Stefanie Giebert (2014) nos da un impulso para la enseñanza de L2 a través del teatro, en adultos profesionales, en su investigación Théâtre et enseignement des langues de spécialité. Si bien el propósito de esta investigación es plantear un programa para uso de la escenificación en el bachillerato, las ideas de Giebert son útiles. Según ella, los profesionales requieren de la apropiación de un juego de lenguaje especializado que puede ser proveído de manera sencilla por medio de situaciones performáticas al generar un contexto adecuado: reuniones, citas de trabajo, cenas de negocios, entre otros. A través de la dramatización, los estudiantes pueden vivenciar (de manera simulada) la importancia y el uso de las emociones en situaciones profesionales reales. Esto es evidente en profesiones de negocios, pero igualmente importante desde el punto de vista desde la asertividad y lo comunicativo en profesiones más técnicas como ciencias e ingeniería. A través del teatro los estudiantes aprenden "a pararse en los zapatos de otro", lo cual es fundamental en la construcción de una identidad abierta y respetuosa con los otros.

La ética laboral de muchas culturas puede ser conocida a través de la representación de obras teatrales. En conclusión, para Giebert la importancia del teatro en el aprendizaje de L2 para bachillerato también es extensible para adultos profesionales, donde las situaciones y los contextos son esenciales para el aprendizaje vital de una lengua. La importancia de esta investigación, para nosotros, estriba en el hecho de que el propósito de un acto pedagógico define sus prácticas. No es lo mismo crear un programa para la enseñanza de L2 en estudiantes del bachillerato que para profesionales especializados. 
Así mismo, los estudiantes más rezagados dentro del grupo experimental tienen mayor riqueza de vocabulario y mayor memorización del lenguaje usado, a través de las actividades de dramatización, que los mejores estudiantes del grupo control. Esta es de las investigaciones que hacen énfasis tanto en las actividades a desarrollar, como en la parte evaluativa, lo que será de vital importancia para la presente investigación.

Evelyn Gualdrón y Etna Castillo (2018) son las responsables del grupo $E$ Theater, un grupo interdisciplinario que existe en la Universidad Nacional de Colombia, donde utilizan el teatro para la enseñanza de L2. Ellas documentaron su experiencia constante por más de ocho años en el artículo Theater for Language Teaching and Learning: The E Theater, a Holistic Methodology. Allí, ellas insisten en que el teatro desarrolla varias inteligencias múltiples, así como también permite la inmersión en un ambiente donde el error y la equivocación dejan de ser un riesgo para que el aprendiz pueda acostumbrarse a él, siempre que no haya un director impositivo. Es así como surge unos de los conceptos más importantes de su aporte: el filtro afectivo. Según ellas, hay una parte emocional del aprendizaje que se puede disminuir o potencializar de acuerdo con el ambiente, según el estudiante esté expuesto a los demás. Esto es análogo a lo que sucede con un actor ante su audiencia. De esta forma, la maximización de la motivación del aprendizaje no se logra no exponiendo al estudiante, sino dotándolo de los medios para que afronte situaciones de exposición y salga bien librado. Cuando el estudiante enfrente de forma gradual distintos filtros afectivos, adquirirá las competencias comunicativas que han sido descritas por otros investigadores.

\section{INTERVENCIÓN}

El English Day en el Colegio Gimnasio Los Alerces es considerado un estudio de caso, dado que se trata de la instanciación de un fenómeno, dentro de una institución (como lo es la escuela), que se puede controlar, observar y repetir, en los términos que plantean Sehaub y Zenke (2001), Arzaluz (2005) Díaz, y Mendoza y Porras (2011). Se recolectó la información por medio de registro fílmico, fotográfico y una encuesta. Los resultados, se enuncian por medio de (1) una triangulación metodológica (O'Donoghue y Punch, 2003), (2) al final de esa sección hay un análisis y una descripción estadística del instrumento. Y (3) una propuesta conceptual, hecha con base en la interpretación de las investigaciones sobre la enseñanza de L2 y el caso estudiado.

\section{- Población}

La actividad cuyos resultados se muestran y analizan en este trabajo está diseñada para y por estudiantes del grado octavo, y el profesor Alejandro Bedoya, del Colegio Gimnasio los Alerces, ubicado en el barrio Villa Luz, en la localidad de Engativá, ciudad de Bogotá, departamento de Cundinamarca, Colombia-Sur América. El rango de edad de los estudiantes va desde los 13 a los 15 años. Hubo un total de 14 participantes, 3 mujeres y 11 hombres. El nivel de inglés es bajo, razón por la que la institución decidió emprender iniciativas y alternativas que aumentara el rendimiento en el aprendizaje de L2.

\section{- $\quad$ Estrategia lúdico - didáctica "estudio de caso. English Day. Gimnasio Los Alerces. Grado octavo.}

El English Day es una actividad que se hace en algunos colegios de Colombia. Ella consiste en un día al año en el que se llevan a cabo varias actividades performáticas en inglés, para que sean disfrutadas por los estudiantes, profesores, 
directivas y padres de familia que hacen las veces de espectadores. En ocasiones se hace dentro del marco del día del idioma, resaltando que no solo el español es nuestro puente de comunicación entre culturas, sino que el inglés juega un papel esencial en la comunicación del mundo contemporáneo. En algunas instituciones se celebra dentro de las izadas de bandera y, en otras, se trata de una celebración de un día particular y especial. A nuestro juicio, es la figura que institucionaliza en los colegios de Colombia las prácticas del uso del drama como estrategia didáctica para la enseñanza de L2. Se trata de una fecha contemplada desde el final de un año lectivo para la planeación del calendario del año siguiente. Esto, a su vez implica que el diseño y la ejecución de las actividades entran en el registro que tienen las secretarías de educación del país, sobre la planeación curricular de un colegio.

Este proceso, proyecto y actividad tiene todo el respaldo institucional y, organizado por los docentes de L2, implica varios componentes teóricos, conceptúales, prácticos y otros, expuestos en el marco teórico. En particular, en el Gimnasio Los Alerces, el último English Day se celebró el día 25 de octubre de 2019. El colegio cuenta con un proyecto de enseñanza de L2 por niveles: inicial, intermedio y avanzado. Para cada uno de esos niveles hay un profesor asignado. Cada año, todas las actividades del English Day, para todos los cursos, gira en torno de una temática específica que es escogida por los profesores del área.

- Propósito: Crear un contexto sociohistórico cognitivo y axiológico de acercamiento propicio donde los estudiantes logren tener un proceso de inmersión facilitador del aprendizaje de L2 a través del montaje de teatro.
Paso 1. Directriz institucional de tratar temas históricos en la jornada del English day.

Paso 2. Lluvia de ideas para escoger el tema histórico. Se escogió Al Capone.

Paso 3. Escritura grupal del guión de un sketch llamado Al Capone, a cargo de los estudiantes del grado octavo, bajo la supervisión del profesor. Texto de cinco páginas y montaje de 22 minutos.

Paso 4. Asignación o creación de personajes para cada estudiante. Para las estudiantes mujeres se utilizaron canciones ambientadas en los años 20, 30 y la puesta en escena fue complemento vital del guión. Un total de tres canciones.

Paso 5. Ejercicios de improvisación individuales para cada estudiante con el fin de apropiarse del personaje.

Paso 6. Ensayos colectivos de la obra.

Paso 7. Puesta en escena del sketch Al Capone en el English day.

- $\quad$ Aplicación de encuesta en el análisis y la descripción a partir de conceptos y la experiencia

La puesta en escena del grado fue celebrada por directivas, profesores y estudiantes. La didáctica de la escenificación para la enseñanza de L2 contó con muy buen pre-diseño y diseño instruccional. En la encuesta aplicada a estudiantes fue posible visualizar que la obra tuvo un impacto positivo sobre el grupo. A continuación, la triangulación de resultados:

\footnotetext{
- $\quad$ Pasos para el montaje de la obra
} 


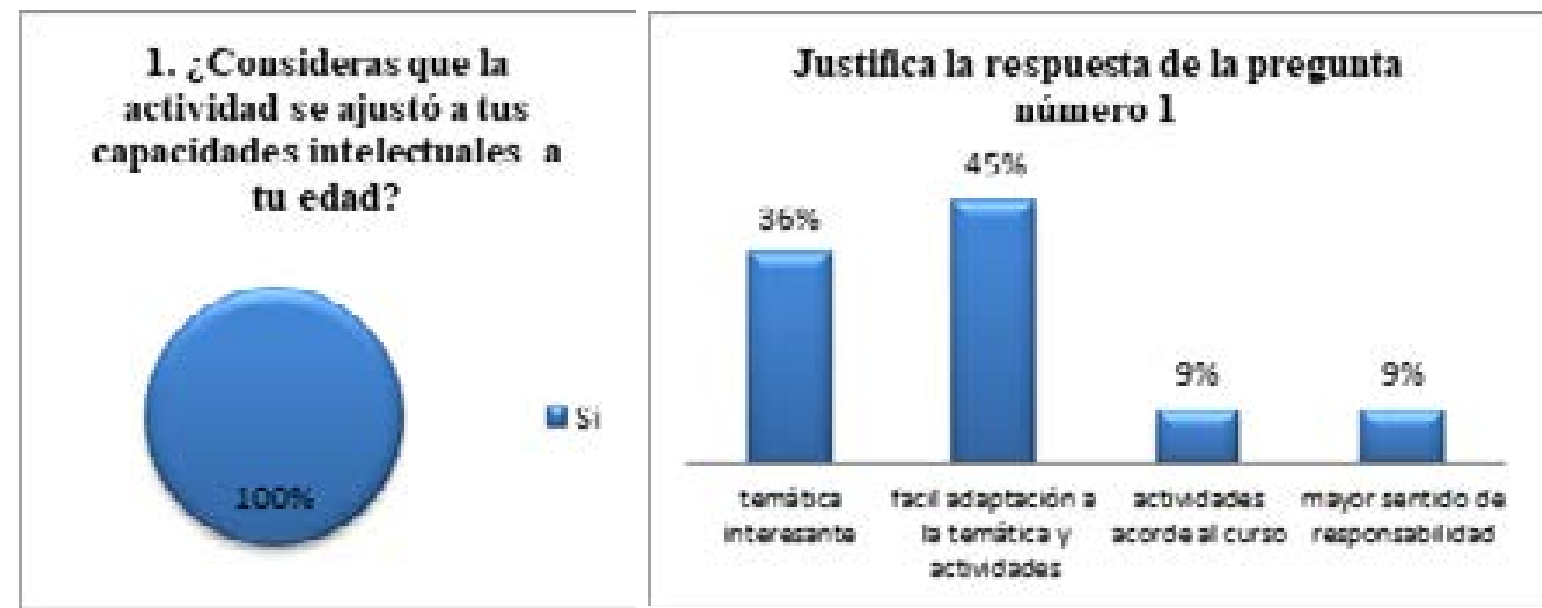

Gráfica 1. Pregunta número 1. Fuente: elaboración propia.

La gráfica 1 refleja frente al ejercicio abordado, el reconocimiento de los procesos y que la didáctica está diseñada acorde con sus capacidades; dentro de los aspectos que sobresalen, los estudiantes reconocen que la temática fue fácil de abordar y pudieron adaptarse fácil a las actividades. Así mismo, varios estudiantes se sintieron atraídos por el tema de la obra, lo que permitió que se mantuvieran interesados en la didáctica. Esto corrobora la importancia de definir la población a la que se dirige un acto pedagógico, según Giebert (2014).

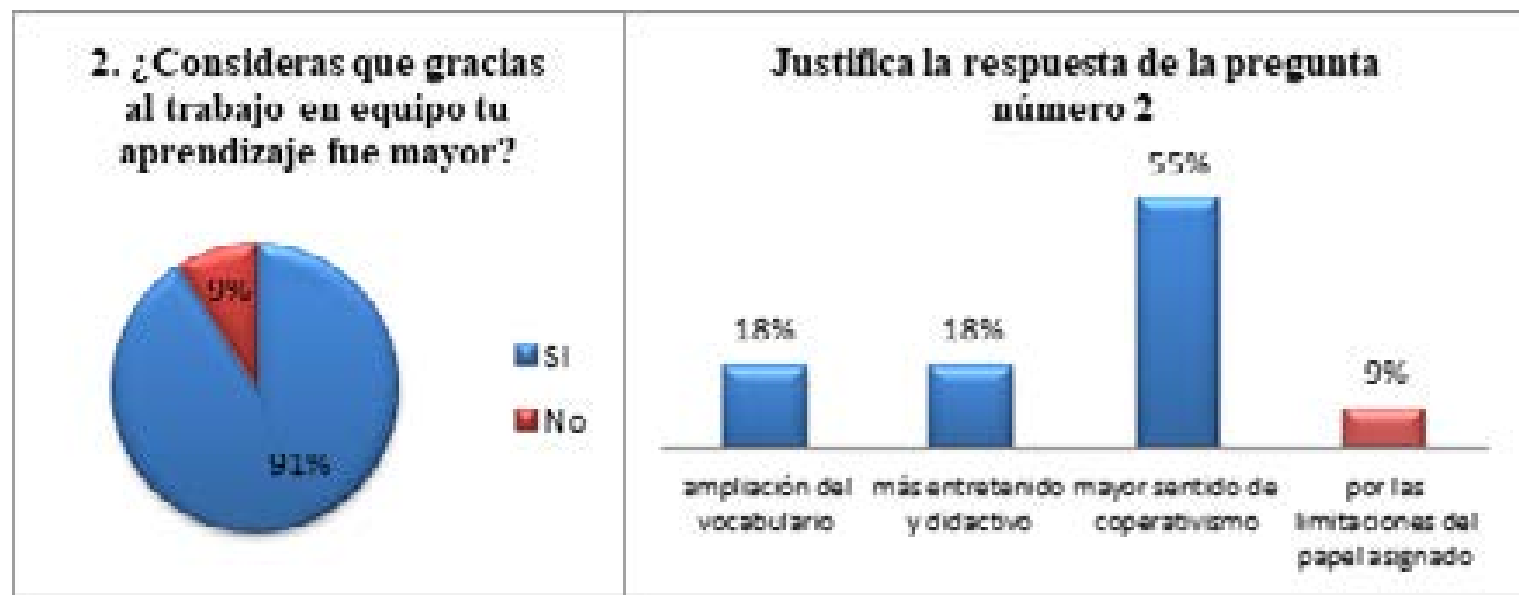

Grafica 2. La pregunta dos evidenció un alto grado de conformidad con el trabajo en equipo. Pregunta número 2. Fuente: elaboración propia.

Dentro de los aspectos más significativos de la experiencia, destacan un mayor sentido de cooperativismo, pues los estudiantes mencionan cómo, entre todos, apoyaron a quienes tenían alguna falencia y lograron fortalecerlas. Por otro parte, un pequeño grupo de estudiantes se desmotivó, dado que su personaje no representó un papel importante en la obra, al punto de considerar que su aprendizaje fue menor al de los otros compañeros con asignación de personajes más dinámicos e interesantes. 


\section{3. ¿Qué tanto consideras que la experiencia de construir un libreto en el Euglish Day contribuyo al mejoramiento del vocabulario $y$ la gramática en inglès?}

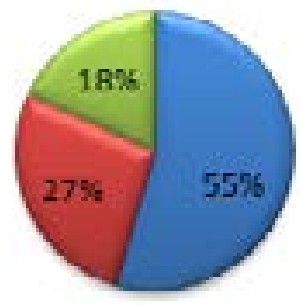

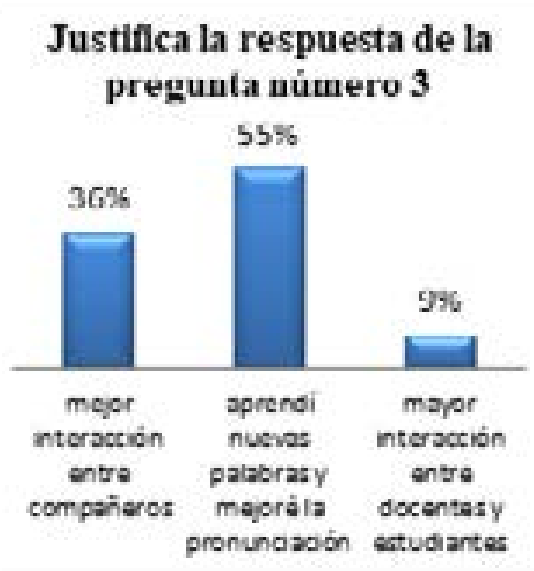

Gráfica 3. Pregunta número 3. Fuente: elaboración propia.

Las respuestas a la pregunta tres muestran que, si bien un pequeño porcentaje de estudiantes considera la experiencia como algo regular, más del $80 \%$ afirma que contribuyó bastante al mejoramiento gramatical y de vocabulario; cabe resaltar que, en la justificación de las respuestas a la pregunta 3 , todos los encuestados opinaron de forma positiva acerca de la obra de teatro.
Entre lo más destacado, el grupo de estudiantes reconoció que el ejercicio le permitió aprender nuevas palabras y mejorar la pronunciación; de nuevo, varios de ellos resaltan el trabajo en equipo como parte fundamental del mejoramiento en el vocabulario y la gramática del inglés. Esto corrobora lo dicho por McGovern (2017) sobre la creación de ambientes propicios a través del drama para el aprendizaje de L2.

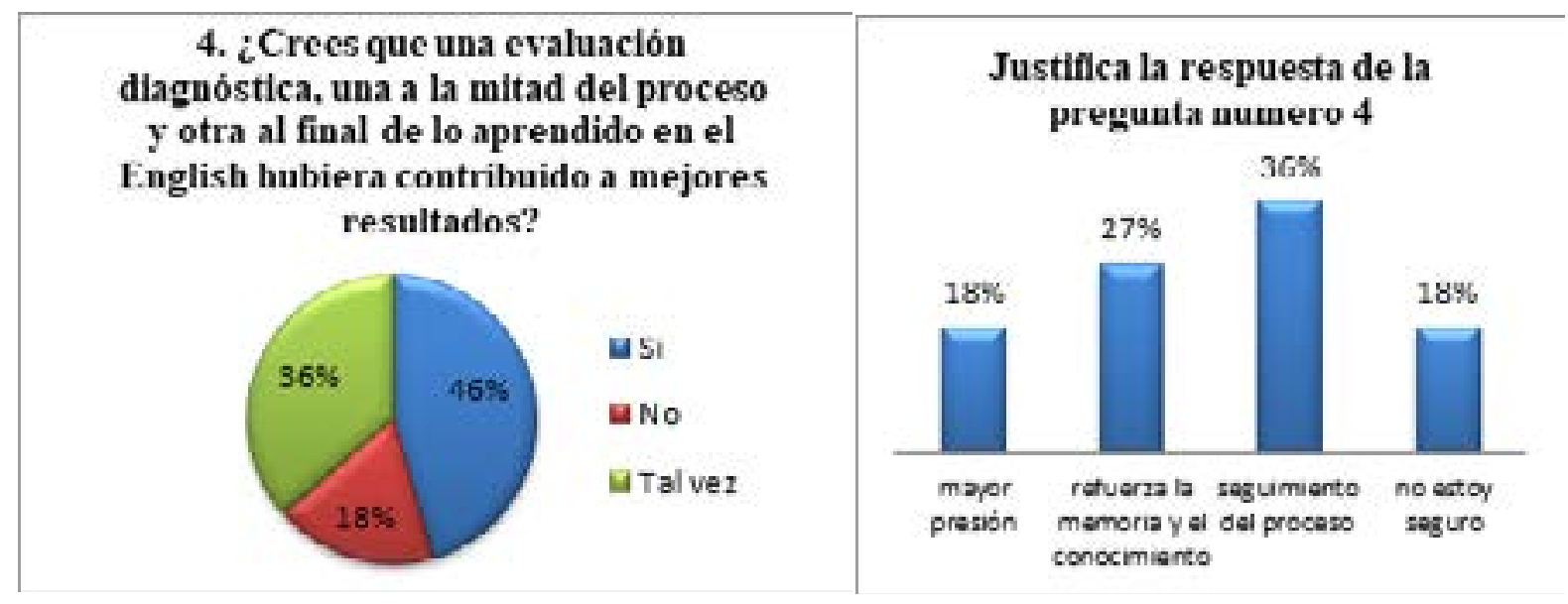

Grafica 4. Pregunta número 4. Fuente: elaboración propia.

A la pregunta sobre una evaluación diagnóstica, otra intermedia y una más al final del proceso, cerca del $50 \%$ de los estudiantes consideran que dicho diagnóstico sí puede contribuir a mejorar resultados. Dentro delos motivos más destacados es que puede ser una buena herramienta para hacer un adecuado seguimiento del proceso de enseñanza-aprendizaje, al mismo tiempo que refuerza la memoria y los conocimientos; por su parte, la otra mitad del grupo parece no estar de acuerdo con o no sentirse seguro de que sea una buena estrategia, pues varios consideran que puede generar mayor presión, lo que se reflejaría en un menor rendimiento en las actividades. Sin embargo, se considera que esto se debe a que prima una un prejuicio 
sobre la concepción tradicional de la evaluación y su supuesto carácter punitivo, y no atribuirle un carácter más del lado de la verificar si se logra un propósito. Creemos, con Gabitova, Shayakhmetova y Beisembayeva (2018), este elemento pedagógico merece una mayor atención. Estamos seguros que al recurrir a evaluaciones de tipo activo, donde se aprende y se aprehende al tiempo que se evalúa, estos prejuicios desaparecerán.

Tabla 1.

Pregunta número 5.

\begin{tabular}{l} 
5. ¿Qué enseñanzas acerca de cumplir o infringir la ley aprendiste a través de la obra sobre \\
Al Capone, en el English Day? \\
\hline $\begin{array}{l}\text { Que absolutamente todo lo que hacemos tiene consecuencias, así nos hayamos arrepentido } \\
\text { después de hacerlo. }\end{array}$ \\
\hline $\begin{array}{l}\text { Que en cierta medida se sentía bien, pero al final te das cuenta que realmente no sirve de nada } \\
\text { porque te está persiguiendo medio país. }\end{array}$ \\
\hline $\begin{array}{l}\text { Que todo se paga en la vida, un ejemplo de esto es Alcapone, el murió triste y solo en la cárcel } \\
\text { pagando por todas las atrocidades que hizo. }\end{array}$ \\
\hline $\begin{array}{l}\text { "Ser correcto y nada problemático" fue la enseñanza, ver las consecuencias que se pueden crear } \\
\text { más adelante y el cómo te puede destruir el infringirlas o ayudar el cumplirlas. }\end{array}$ \\
\hline Que al cumplir las leyes tenemos una vida más tranquila y sin preocupaciones. \\
\hline De no vender trago. \\
\hline $\begin{array}{l}\text { Aprendí que todo lo que hacemos tiene una consecuencia bien sea mala o buena, así que } \\
\text { siempre debemos de pensar dos veces antes de cometer algún delito. }\end{array}$ \\
\hline Que la "mafia" desde siempre ha existido y siempre termina mal. \\
\hline Debemos respetar la ley o podremos acabar muy mal. \\
\hline Que debemos cumplir con la ley para no llevar una mala vida ni una vida de riesgos. \\
\hline $\begin{array}{l}\text { Toda persona no digna de ser un buen ciudadano, siempre tiene cosas buenas, en el salón } \\
\text { quedamos sorprendidos cuando al Capone fue el responsable de los primeros comedores } \\
\text { sociales en Estados Unidos. }\end{array}$ \\
\hline
\end{tabular}

Nota: La pregunta se formuló con el fin de determinar si el ejercicio había permitido una buena comprensión temática por parte del estudiantado. Fuente: elaboración propia. 
Los enunciados de las respuestas se describen en la tabla 1. Se puede afirmar que el ejercicio permitió una buena comprensión temática de la obra escogida y, en adición, el fortalecimiento y la formación de valores sociales entre el estudiantado, en consonancia con lo que mostraron. Esto respalda lo planteado por Paul (2015) y Gualdrón y Castillo (2018) cuando resaltan la utilidad del teatro para la expresión de emociones y el aprendizaje de componentes axiológicos de las culturas que hablan la lengua aprendida, en este caso, el inglés. Si hacemos énfasis en el aprendizaje de valores sociales, la enseñanza de L2 se convierte en una herramienta que conecta a las personas por medio de la eticidad.

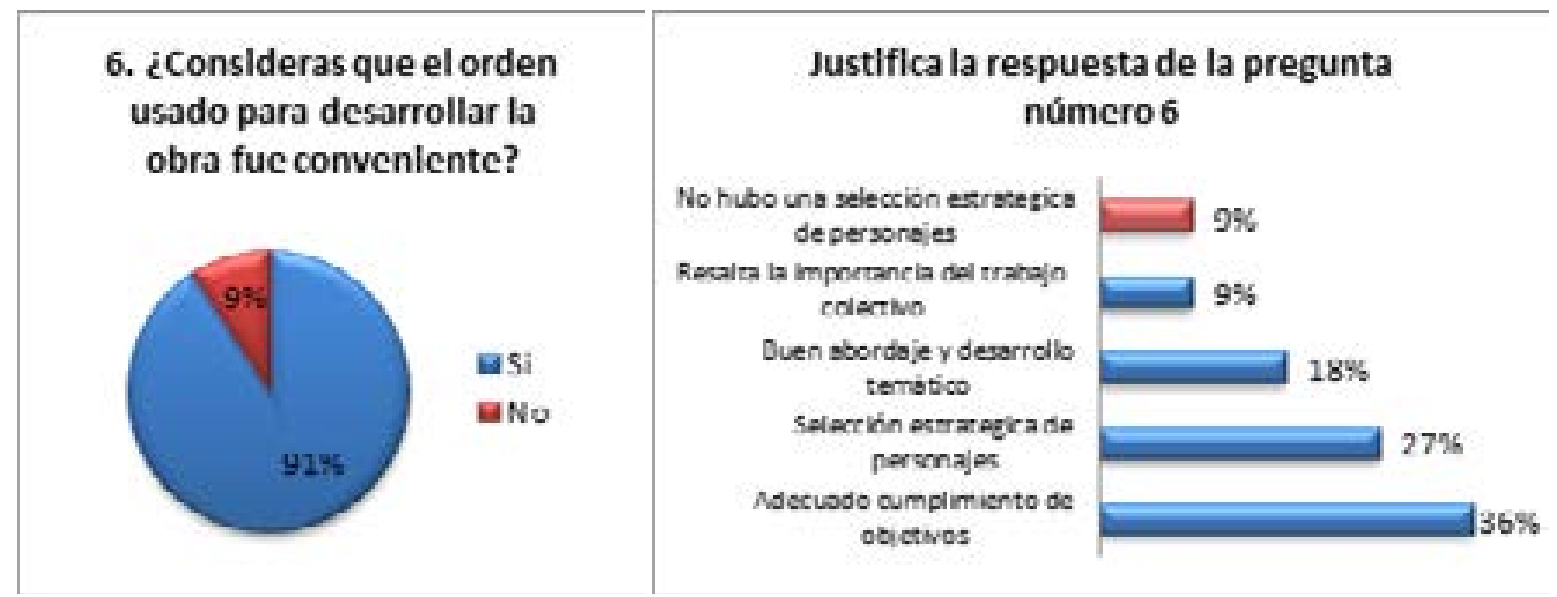

Gráfica 5. Pregunta número 6. Fuente: elaboración propia.

Como lo evidencia la gráfica más del $90 \%$ de los estudiantes se sintieron conformes con la secuencia didáctica escogida para la preproducción y la producción de la obra, un alto porcentaje de ellos consideró que esta estrategia permitió un adecuado cumplimiento de objetivos y se sintieron satisfechos con ello; lo que corrobora la importancia de diseñar las secuencias de enseñanzas, como lo plantean Gualdrón y Castillo (2018).

Por otra parte, varios estudiantes reportaron que el mecanismo para la selección de personajes fue adecuado, ya que permitió que la mayoría se sintiera a gusto con su representación. En este punto, un pequeño porcentaje resalta en este punto cómo la estrategia permitió reconocer la importancia del trabajo colectivo. Solo un $9 \%$ de los estudiantes se sintió inconforme con este aspecto dentro de la estrategia y manifestó que el profesor debió elegir a los estudiantes que debían interpretar cada personaje, dada su experiencia y conocimiento de las diferentes aptitudes en el grupo. En todo caso, consideramos para el programa sugerir que la decisión sea mancomunada entre profesor y estudiante para construir mejor los ambientes de los que hablan Gualdrón y Castillo (2018).

Así se triangulo el trabajo etnográfico en el estudio de caso hecho por el autor, la revisión documental de las concepciones del uso del teatro-drama-performance para la enseñanza de L2 y la encuesta a los estudiantes, para cumplir con lo propuesto por O'Donoghue y Punch K. (2003). 


\section{CONCLUSIÓN}

Los elementos, o momentos, que conforman la estructura de todo acto pedagógico son los propósitos, las enseñanzas, secuencias, didácticas, recursos y sistema evaluativo (De Zubiría, 1997). De la misma forma, el desarrollo de un programa educativo debe estar previsto en tres dimensiones humanas: cognitiva, axiológica y práxica (De Zubiría, 1997). Con base en estas ideas y de todo lo que se ha puesto de manifiesto en el presente artículo, le proponemos a los docentes del país las siguientes consideraciones:

\section{FUNDAMENTOS Y PROPÓSITOS}

Para el uso del performance en la enseñanza aprendizaje de L2 es fundamental que el docente tenga en cuenta, antes que nada, lo propósitos. De acuerdo con la revisión documental, estos se entienden de dos maneras: pedagógicos y didácticos. La diferencia entre la pedagogía y la didáctica es la concepción antropológica y política. La pedagogía se pregunta por los propósitos finales de educar a un ser humano, ¿qué tipo de ser humano ha de entregársele a la sociedad? (De Zubiría, 1997). La didáctica se pregunta sobre cómo enseñar, bajo qué métodos. En este orden de ideas, la didáctica solo tiene sentido dentro y por una concepción pedagógica. De acuerdo con lo anterior, los propósitos pedagógicos de usar el performance para la enseñanza de L2 son axiológicos, sociocognitivos y práxicos.

\section{PROPÓSITOS PRÁXICOS.}

La concepción antropológica que subyace al uso del performance en L2 es perfectamente recogida por la escuela de Chicago (Paul, 2015), según la cual el ser humano es performático por naturaleza. Todos los seres humanos tienen la capacidad de actuar y también la necesidad, si entendemos actuar en relación con el juego, la simulación y la puesta en escena indispensables para todo proceso de socialización. Aunque este rasgo antropológico está expresado desde el punto de vista del teatro, ya Huzinga (2007) nos señala el papel del juego como mecanismo de humanización. En este sentido, lo que quiere expresar la Escuela de Chicago es la fuerte conexión entre lúdica y puesta en escena. Acá también hay un eco de la frase de Shakespeare que sirve de epílogo para este artículo. Resulta que la frase no es anecdótica, sino que expresa un rasgo antropológico.

\section{PROPÓSITOS SOCIO-COGNITIVOS.}

Los propósitos de usar el performance en L2 no ha sido enseñar L2. Esto suena paradójico. No se enseña lengua extranjera para aprender lengua extranjera, se enseña para transmitir una serie de códigos que contienen la historia, los valores, las creencias, las expectativas y las experiencias de una cultura ajena que, quizá, solo se puedan entender lo mejor posible si se hace mediante los códigos en los que se transmitió la experiencia original. Dicho en términos filosóficos, se trata de entrar en contacto con el Otro, cuando ese otro forma parte de una cultura diferente a la nuestra.

\section{PROPÓSITOS AXIOLÓGICOS.}

Desde algunos puntos de vista o percepciones, el propósito de la enseñanza de L2 tampoco es que el estudiante aprende L2. Ha sido la asimilación de valores individuales (sin desmedro de su importancia social) que contribuyan a la dignificación de él y los otros cercanos: confianza, trabajo en equipo, cooperación, solidaridad. En este sentido, el docente puede tener en cuenta las características de su grupo particular e incluir en la parte axiológica de su programa, aquellos valores que hacen falta desarrollar en los estudiantes, así como también aquellos que son más acentuados y valga la pena destacar. 
Dentro de los propósitos didácticos a tener en cuenta es la población a la cual se diseña la actividad, el rango de edades y un perfil social, para cumplir con las indicaciones que dan Giebert (2014) y (Paul, 2015).

\section{ENSEÑANZAS}

Blandas: Las habilidades que han enmarcado el uso del performance para L2 han sido la oralidad, la expresividad, el manejo de público, la memoria creativa y corporal, así como también la autogestión en términos de adquisición de contenidos específicos con la generación de ambientes propicios McGovern (2017) y Gualdrón y Castillo (2018).

Contenidos: tienen que ver con conceptos y estructuras propias de una lengua. Se trata de la parte gramatical necesaria para tener un código de comunicación estándar, desde el punto de vista descriptivo. Un prejuicio generalizado relega a la gramática como innecesaria a la hora del aprendizaje de una segunda lengua, desde que surgieron los enfoques comunicativos. Lo que se puede concluir del estado del arte y de la experiencia en Los Alerces, es que nunca se ha pretendido relegar a la gramática, sino a su aprendizaje a través de la memoria evocativa como fin último. La gramática es un medio y su enseñanza no puede ser anquilosada. Por lo demás, es necesaria en su justa proporción.

\section{SECUENCIAS}

Se entiende por secuencia didáctica el orden en que se lleva a cabo la instrucción con el fin de que los propósitos pedagógicos se cumplan. En la revisión de la literatura, se identificó una actitud bastante abierta al momento de definir este orden, lo que se traduce en que ningún autor propone una secuencia rígida, sino una especie de menú en el que el docente puede escoger, según su criterio.
La primera empieza con los ejercicios de improvisación y monólogos propios de las didácticas performáticas. Siguen los ejercicios propios de la escritura colectiva de guiones donde la adaptación, la asignación y creación de personajes tienen lugar. En un tercer momento vienen los ensayos $y$, al final, la puesta en escena de un proyecto común (Gualdrón y Castillo, 2018).

Pese a lo anterior, observamos que el mismo criterio subyace en la preocupación de los investigadores consultados: la primera estrategia a la que ellos recurren en su secuencia didáctica es la motivación, tanto individual, que se traduce en la disposición, como en la grupal, lo que implica la creación de un ambiente, una situación, un momento propicio para el aprendizaje. Este ambiente sirve como catapulta para que la situación performática, que implica dejar la pena y los prejuicios a un lado, tenga lugar y el aprendizaje se dé sin problemas. Un segundo presupuesto es aprovechar ese ambiente para que los estudiantes cometan errores. En ese sentido, la situación propicia se usa para que los aprendices aprendan mediante el error, lo que exige que ellos experimenten momentos de motivación maximizados. Un tercer criterio es la adquisición de contenidos específicos, que de alguna forma son necesarios para la comunicación mediante una lengua. La recomendación para los docentes es que definan la secuencia de enseñanzas sin olvidar que el propósito central debe ser que los estudiantes cometan la mayor cantidad de errores posibles que se corrijan y retroalimenten en un ambiente favorable.

Con base en lo anterior, y en la experiencia de Los Alerces, sobre algo que no se vio muy reflejado en la literatura revisada, acá se plantea empezar con requerimientos que impliquen la participación de los estudiantes en todo momento, desde el inicio. Esto sirvió mucho para despertar y maximizar la motivación de ellos. 


\section{DIDÁCTICAS}

Este es el elemento curricular del que mayor consciencia se tiene en la enseñanza de L2 a través de la escenificación, según se vio en la revisión documental. Consta de los tres componentes identificados por McGovern (2017):

Didácticas para la gramática en uso y contexto: aquí se ubica la escritura del guión, y afines, que se recomienda llevar a cabo de manera grupal, para aprovechar el ambiente que se genera al aprender unos estudiantes de otros y la retroalimentación que, entre ellos, puedan darse. La gramática es una serie de reglas. Por eso su aprendizaje requiere la asimilación de un código. Sin embargo, la construcción colectiva de un libreto, como se evidenció en la experiencia de English Day para el grado octavo, contribuyó a la construcción de un ambiente propicio para el aprendizaje de la gramática y la memorización performática. Mientras el texto era compuesto por los estudiantes, ellos recibían retroalimentación del docente en las formas "correctas" del inglés, con la ventaja de que el propósito de la actividad no era la gramática, sino que la gramática era el medio para que ellos pudieran recrear una situación. Recomendamos ampliamente este enfoque por permitir el desarrollo de las competencias comunicativas dentro de un ambiente favorable.

Didácticas para el aprendizaje individual a través del error: En la segunda, están los monólogos y los ejercicios de improvisación, donde se construyen personajes o se tratan temas puestos en escena con una carga directriz muy fuerte por parte del aprendiz. Por ello, exigen un esfuerzo auto-estructurante. La puesta en escena de la obra es la excusa perfecta para un esfuerzo hetero-estructurante, lo que sin embargo implica un esfuerzo más que nosotros queremos proponer: una serie de didácticas en torno al diseño del propio proyecto de puesta en escena. Esto quiere decir, el esfuerzo por participar en la creación colectiva de la actividad implica conocimientos en torno a diseñar esas actividades. Esto lo dejamos para ser considerado por los docentes.

En los ensayos se pudo evidenciar la segunda aproximación performática de la que habla McGovern (2017) que son las improvisaciones. Muchos de los personajes finales de la obra empezaron a construirse a partir de monólogos, que requerían ejercicios de improvisación. Cada estudiante debía dar a su personaje un poco de su carácter, hasta que tuviera "vida propia". Para ello se usaron los ejercicios de improvisación.

Didácticas para el trabajo en equipo: La puesta en escena final, que implica al cuerpo y la exposición frente al otro constituye la tercera aproximación de McGovern (2017). En cuanto a esta parte, la conclusión es que hay un mejor desempeño en la escenificación, mientras se logre un mejor equilibrio entre la apropiación de los elementos de L2 y las capacidades histriónicas y actorales. Apuntar a ese equilibrio es lo que debe hacer cualquier programa para el uso de la escenificación como didáctica para L2.

La actividad mostró que la combinación de los tres enfoques didácticos compensa lo que cada uno por separado no puede ofrecer: errores gramaticales se corrigen en la puesta en escena. La apropiación y personificación de valores incentiva el aprendizaje. La participación en una obra afianza la autoconfianza, lo que será útil en cualquier empresa en la vida de los estudiantes.

Si bien, en el prediseño se dijo que la mayor parte del guión fue escrito por pocos, también sucedió que la repetición y la corrección del mismo guión, durante los ensayos, sirvió para llevar a cabo una especie de aprendizaje colectivo donde el grupo terminó memorizando los parlamentos de todos los implicados, de manera tal que cuando algún estudiante hizo falta en el ensayo, otro pudo relevarlo. Esto refleja el ambiente propicio 
para el aprendizaje del que hablan Gualdrón y Castillo (2018).

\section{RECURSOS}

Se entiende por esa palabra a los medios físicos y abstractos a través de los cuáles se entrega una enseñanza (De Zubiría, 1997). El sentido común casi nos obliga a distinguir recursos físicos de recursos humanos. En ese sentido, cada uno de los participantes en la actividad suma un papel crucial en el desarrollo de la misma. Queremos, poner, sin embargo, en consideración algo que se reflejó en el caso de Los Alerces:

La falta de espacio en las instalaciones del colegio y de presupuesto económico jugó un papel clave a la hora de poner en escena el sketch. No es posible contar con recursos de los estudiantes o sus padres y las instituciones en Colombia no cuentan con presupuestos para montajes teatrales. Esto, en lugar de ser un problema, termina siendo una fuente de motivación y activación para la creatividad: debido a que la obra transcurría en cuatro lugares, la mesa que se usó terminó resignificando el espacio y siendo una especie de protagonista. Se trataba de la mesa que servía para que la madre de Al Capone sirviera los alimentos al regañarlo. Al retirar el mantel, la mesa mostraba una cobertura verde para los juegos de billar. Al retirar ese tapiz verde, la mesa posibilitaba la reunión de los gansters para transportar a los espectadores a un bar. De la misma manera, los espacios reducidos condicionaron las actuaciones, de forma que los estudiantes tenían que representar sus papeles simulando la disminución de la velocidad, como en cámara lenta, para que los espectadores vieran bien cada actuación. Es por lo anterior, que invitamos a jugar con los recursos a disposición de manera creativa, tanto para abordar la relación entre el cuerpo y el espacio, como para resignificar los objetos en la obra.

\section{EVALUACIÓN.}

Se recomienda una evaluación diagnóstica del nivel de inglés de los estudiantes en las competencias escrita, oral, de escucha y lectura. De esta manera se tiene un punto de partida. Recomendamos que el diseño del sistema evaluativo posterior se haga con base en la secuencia didáctica que se escoja. Así se puede contemplar una evaluación para la parte gramatical en uso y contexto. Esto implica evitar al máximo que los requerimientos de la evaluación pasen por la memoria evocativa, y promulguen el uso de la memoria creativa. Por otro lado, las observaciones dirigidas durante los ejercicios individuales de improvisación y monólogos son de utilidad para ver la mejora en el vocabulario y la mejora en la capacidad de expresarse en público. Se requiere también tener en cuenta la mejora grupal, sobre todo en las actividades de construcción del guión y en los ensayos. No está demás, basarse en las consideraciones que tienen los compañeros entre ellos, para generar otro criterio. Así, podemos resumir de la siguiente forma:

Fase 1: evaluación diagnóstica cuantitativa.

Fase 2: evaluación individual observacional.

Fase 3: evaluación grupal de ítems cognitivos y expresivos.

Fase 4: evaluación final para contraste con el diagnóstico.

A lo sumo, dado todo el desarrollo pedagógico mostrado hasta acá, sostenemos que evaluar la eficiencia del uso del performance para el proceso de enseñanza aprendizaje requiere de un sistema evaluativo multifactorial 


\section{REFERENCIAS}

American Phycology Association (2017). Manual de publicaciones (Trad de Guerra, M). México D. F., México: Manual Moderno.

Arzaluz, S. (2005). La utilización del estudio de caso en el análisis local. Región y sociedad, 17(5) 107-144.

Blanco, P. (2001). El teatro de aula como estrategia pedagógica. Proyecto de innovación e investigación Pedagógica. Recuperado de: http:// www.cervantesvirtual.com/obra-visor/ el-teatro-de-aula-como-estrategiapedagogica-proyecto-de-innovacione-investigacion-pedagogica-- $0 /$ html/0023cd44-82b2-11df-acc7002185ce6064_2.html

Corral, A. (2012) El teatro en la enseñanza de lenguas extranjeras. La dramatización como modelo y acción. Didáctica, lengua y literatura. doi: 10.5209/rev DIDA.2013.v25.42238

De Zubiría, Miguel. (1997). Pedagogía Conceptual: desarrollos filosóficos, pedagógicos y psicológicos. Bogotá, Colombia: Fundación Alberto Merani.

Danzi, D. (2016). El teatro para el aprendizaje de idiomas. En Primer congreso internacional sobre psicología y pedagogía del teatro. Recuperado de: http://webs.ucm.es/BUCM/revcul/elearning-innova/194/art2779.pdf

Day, R. (2005) Cómo escribir y publicar trabajos científicos. (Trad de Sáenz, M). Washington, Estados Unidos. Oficina Regional de la Organización Mundial de la Salud.

Díaz, S., Mendoza, V., y Porras, C. (2001) Una guía para la elaboración de estudios de caso. Razón y palabra, 1(75), 1-26.

Gabitova, L., Shayakhmetova L., Beisembayeva, Z. (2018). The effectiveness of drama methods in the development of communication skills. Revista Publicando. 5(16), 308-315. Recuperado de https://revistapublicando.org/ revista/index.php/crv/article/view/1501/ pdf_1102

Giebert, S. (2014). Drama and theatre in teaching foreign languages for professional purposes. Langues de spécialité et professionnalisation, 33(1). doi: 10.4000/apliut.4215

Guzmán, V. (2012). Teoría Curricular. Tialnepantla, México. Red Tercer Milenio. Recuperado de http://www.aliat. org.mx/BibliotecasDigitales/Educacion/ Teoria_curricular.pdf

Gualdrón, E., y Castillo, E. (2018). Theater for language teaching and learning: The $\mathrm{E}$ Theater, a holistic methodology. Profile: Issues in Teachers' Professional Development, 20(2), 211-227. doi: 10.15446/profile.v20n2.63969.

McGovern K. (2017). Conceptualizing Drama in the Second Language Classroom. In: Scenario. 11(2) 54-62. Recuperado de https://www.researchgate. net/publication/333381372 Conceptualizing_Drama_in_the_ Second_Language_Classroom

Paul, Abigail (2015): Incorporating theatre techniques in the language classroom. Scenario. 9(2), 115-124. Recuperado de http://research.ucc.ie/scenario/201502/08-Paul-2015-02-en.pdf

O'Donoghue, T., Punch K. (2003). Qualitative educational research in action: Doing and reflecting. London: Routledge. 
Recuperado de https://epdf.pub/ qualitative-educational-research-inaction-doing-and-reflecting.html

Ronke, A. (2005) Wozu all das Theater? Drama and Theater as a Method for Foreign Language Teaching and Learning in Higher Education in the United States (Tesis doctoral). Universidad de Berlin, Alemania. Recuperado de https://www.depositonce.tu-berlin.de/ bitstream/11303/1448/1/Dokument_8. pdf

Santaella, M. (2006). La evaluación de la creatividad. Sapiens, 7(2), 89-106. Recuperado de https://www.redalyc.org/ pdf/410/41070207.pdf

Schaub H y Zenke K. (2001). Diccionario Akal de pedagogía. Madrid, España: Akal ediciones.

Villagrán, A. y Harris, P. (2009). Algunas claves para escribir correctamente un artículo científico. Revista Chilena de Pediatría. 80 (1): 70-78. doi: 10.4067/S037041062009000100010 